\title{
Models of adaptation of the milking machines systems
}

\author{
Vasyl Dmytriv ${ }^{1, *}$, Ihor Dmytriv $^{1}$, Yurij Lavryk ${ }^{1}$, and Ivan Horodeckyy ${ }^{1}$ \\ ${ }^{1}$ Lviv National Agrarian University, Faculty of Mechanic and Power Engineering, Lviv-Dubliany, Ukraine
}

\begin{abstract}
Two systems of milking machines were considered - biotechnical and vacuum. Methodology for estimation of efficiency of the "machine-animal" biotechnical system was worked out. The dependences of the efficiency parameters of the technical system operation were analyzed. The $K_{M M O}$ operator load factor of the milking machine was proposed. The factor characterizes the technological process of the machine milking. The analytical dependences were worked out for the simulation of the productivity of milking machines and oscillation of the vacuum-gage pressure. As to the simulation results, when the vacuum pipeline diameter was increased the oscillation of the vacuum-gage pressure decreased. The results of analysis and theoretical researches on technological process of the cow machine milking gave a possibility to define the requirements to the improvement of technological process and technical equipment, which will provide the increase of efficiency of the milking systems. Usage of the developed cyber-physical system of the machine milking of cows will increase the productivity of the milking machine in 1.26...1.85 times. At the vacuum gage pressure oscillation of $\Delta p_{v p}=2500 \mathrm{~Pa}$ the suction ability of milking machine will be $\mathrm{E}=4.093 \mathrm{~m} / \mathrm{s}$ accordingly. The defined index of efficiency of the adapted systems functioning of the milking machine is $K_{B T S 2}=55.3$.
\end{abstract}

Key words: milking machine, mathematical model, vacuum, amplitude, load factor.

\section{Introduction}

We will consider the process of cows milking as a biotechnical system of the "person-machine-animal", that does possible the functional ensuring of realization of the genetic potential of the cow productivity with the interaction of objects of this system. Efficiency of functioning of the biotechnical system depends on the parameters of its objects that ensure the quality and efficiency of technological functions.

As a basic performer of the technological process of milking, the milking machine provides adaptation of the technical system to physiology of milk ejection of the cow through vacuum gage pressure with preset parameter and possibility of its adjusting. The milking machine provides also the parameters control and maintenance at the set level during milking of cows. Pulsating air system of milking machine is the peculiarity of unit arrangement that changes the configuration and functional possibilities. Pulsating air system gives an opportunity also to work autonomically, or in composition of the automatic control system of technological process (Industrial Control - IC) of the machine milking. To operate in the composition of IC the milking machine was additionally completed by the measuring device of milk ejection intensity, microprocessor control unit and other elements of electronics $[1,2]$. However, the vacuum gage pressure forming is still one of important factors of the parameters forming directly that does possible adaptation of the "machine-cow" system. The question of parameters adaptation of the milking machine systems was examined by Dmytriv V.T. and other [3-5], where influence of the technological and construction parameters on work mode of systems is analyzed. Also, the stability of vacuum gage pressure was estimated directly by the group of researchers consisting of Pazzona A., Murgia L., Zanini L. et al., depending on the method of adjusting in the vacuum pipeline or in a milking machine. They set that stabilizing of vacuum by the regulator of gravitational type is more dynamic, and the permanent to time is twice more lower from the computer-based system [6]. An analogical conclusion is also in accordance with research results of the regulators of pulsating vacuum gage pressure [7]. In particular Reinemann D.J., Schuring N. and Bade1 R.D. investigated the vacuum gage pressure oscillation depending on: a) configuration of the vacuum and milk hose systems (length of pipelines, hose diameter and other parameters influencing the losses of pressure); b) flowrates of milk in the milk pipeline; c) rates of air movement in the vacuum pipeline. They set that the vacuum gage pressure was raised up as in a vacuum- and milk pipeline, so in the under-teat chamber of the teat cup in the process of diminishing of the intensity of milk ejection [8].

The analysis of research works shows that the grounding of parameters of the effective functioning of the cows milking bioengineering system requires the development of conception and methodology of

Corresponding author: Dmytriv_V@ukr.net 
optimization of the mentioned system parameters and the same for the technical constituent of this system. Optimization of the bioengineering system parameters is also important for the increase of efficiency of milking machines.

\section{Materials and methods}

\subsection{Effectiveness of the "operator-machine- animal" bioengineering system}

The effectiveness of work of the machine milking operator (MMO) was determined by the productivity for the maximum system load. This parameter depends on the $n_{m u}$ quantity of the milking units and the $t_{m}$ duration of the milking of one cow (with consideration of the preparation and final operations $t_{m}=t_{m m}+t_{p-f}$ ), and also the $Z_{l i}$ labour inputs:

$$
W_{m m}=f\left(n_{m u}, t_{m}, Z_{l i}\right)
$$

where: $W_{m m}$ - the productivity of the milking machine, cows/h., $n_{m u}$ - quantity of the milking units, pcs., $t_{m}-$ duration of the milking of one cow, h., $\mathrm{Z}_{l i}-$ labour inputs, MJ.

Effectiveness of functioning of the technical system of milking was estimated by the quality parameter of functioning of the vacuum system, namely the $\Delta p_{V P}$ amplitude of pressure oscillation of the vacuum system of milking machine. The vacuum gage pressure oscillation depends on the $\Pi_{e s}$ parameters of the mentioned technical system elements, the $K_{\text {fes }}$ quality of its interaction and the $Z_{T S}$ energy expense for the system functioning:

$$
\Delta p_{V P}=f\left(\Pi_{e s}, K_{f e s}, Z_{T S}\right),
$$

where $\Delta p_{V P}$ - the amplitude of vacuum gage pressure oscillation, $\mathrm{Pa}, \Pi_{e s}-$ parameters of technical system elements, $K_{f e s}$ - the quality coefficient of the interaction of technical system elements, $Z_{T S}$ - the energy expense for the technical system functioning, MJ.

Effectiveness of the bioengineering system depends on the cow milk ejection that is limited by the pressure oscillation in the vacuum system of the milking machine. The milk yield decrease can be described by the function as a result of the vacuum gage pressure oscillation [9]:

$$
\Delta Q_{M}=Q_{p} \cdot\left[\frac{\varepsilon}{\varepsilon+1} \cdot\left|1-\frac{2 \cdot E_{1}}{3 \cdot J}\right|^{3 / 2}+\frac{1}{\varepsilon+1} \cdot\left(\left|1-\frac{2 \cdot E_{2}}{3 \cdot J}\right|^{3 / 2}-\left|1-\frac{2 \cdot E}{3 \cdot J}\right|^{3 / 2}\right)\right]^{,(3)}
$$

where $\Delta Q_{M}$ - the loss of cow milk yield as a result of the pressure oscillation, $\mathrm{MJ}, Q_{p}$ - the potential of the cow milk yield, $\mathrm{MJ}, \varepsilon-$ the ratio of the pressure oscillation duration to the total milking duration, $E_{1}, E_{2}-$ the suction ability of the milking machine with the modes accordingly $p_{1}=p+\Delta p_{V P} / 2$ i $p_{1}=p-\Delta p_{V P} / 2, p-$ the vacuum gage pressure in the under teat chamber of the teat cup, $\mathrm{Pa}, E$ - the suction ability of the milking machine for the vacuum gage pressure $p$ in the under teat chamber of the teat cup, $J=E \cdot 2 / 3$ - parameter of the milk ejection.

The suction ability of the milking machine was determined according to the described procedure [9]:

$$
E=K_{f} \cdot K_{\mu} \cdot\left(2 \cdot p / \rho_{M}\right)^{1 / 2} /\left(K_{f}+1\right),
$$

where $K_{f}$ - the proportion of phases of the milking machine, $K_{\mu}$ - flow coefficient, $\rho_{M}-$ milk density, $\mathrm{kg} / \mathrm{M}^{3}$.

On the basis of the above mentioned parameter the effectiveness of the milking machine system is expressed by the formula:

$$
K_{B T S}=\frac{1}{Z_{B T S}} \sum_{n=1}^{i}\left(Q_{p}-\Delta Q_{M}\right)
$$

where $Z_{B T S}$ - the energy expense for the functioning of the milking machine system, $Z_{B T S}=Z_{l i}+Z_{T S}$, MJ.

The demonstrated formula (5) presents that effectiveness depends of the parameters of the technical system. The mentioned technical system has to ensure the conditions of the milk ejection from the cow udder according to the lactogenesis physiology demand with the reduction of the MMO labour inputs and also total energy expense lowering for the functioning of the milking machine systems.

Taking into account the order of the preparation and final operations of machine milking one can determine the $K_{\text {on }}$ load factor of the milking machine operator by the formula [10]:

$$
K_{M M O}=\frac{t_{m}+\left(n_{m u}-1\right) \cdot t_{f}}{n_{m u} \cdot t_{p-f}}
$$

where $t_{m}-$ duration of the one cow milking, $\mathrm{t}_{m}=t_{m m}+t_{p-f}, \mathrm{~s}[11], t_{f}-$ duration of the final operations, $\mathrm{s}$.

If the $K_{M M O}>1-$ operator is underloaded, accordingly the milking machine is not overdone on the udder teats, if the $K_{M M O}<1$ - the machine milking operator is overloaded and the schedule of preparation and final operations is not kept, or the milking machine is overdone on the udder teats of cow.

From the equation (6) we shall derive the formula for determining the optimal quantity of the milking machines, taking into account that $t_{p-f}=t_{p}+t_{f}$, $t_{m}=t_{p}+t_{m m}+t_{f}$

$$
n_{m u}=\left[K_{M M O}+\frac{t_{f}}{t_{p}}\left(K_{M M O}-1\right)\right]^{-1} \cdot\left[\frac{t_{f}}{t_{p}}+1\right],
$$

where $t_{p}$ - the duration of the preparation operations by the MMO, s.

Taking into account the average distribution of the duration of preparation and final operations $\left(t_{w}=K_{M M O} \cdot n_{m u} \cdot\left[\bar{t}_{p} \pm S\left(t_{p}\right)+\bar{t}_{f} \pm S\left(t_{f}\right)\right]\right)$ the equation of the productivity of milking machine will have the appearance:

$$
W_{m m}=\frac{3600 \cdot n_{m u}}{K_{M M O} \cdot n_{m u} \cdot\left[\bar{t}_{p} \pm S\left(t_{p}\right)+\bar{t}_{f} \pm S\left(t_{f}\right)\right]+\left(\bar{t}_{f} \pm S\left(t_{f}\right)\right) \cdot\left(1-n_{m u}\right)}
$$


where $\bar{t}_{p}, \bar{t}_{f}$ - the average value of the duration of preparation and final operations of the cow machine milking accordingly, s, $S\left(t_{p}\right), S\left(t_{f}\right)$ - the root-mean-square deviation of the duration of preparation and final operations of the cow machine milking accordingly, s.

\subsection{Amplitude of the pressure oscillation of vacuum system of the milking machine}

The construction of the milking machine can be presented as the functionally finished elements, which are the constituents of the vacuum system of the milking machine (Fig. 1) [12].

Taking into account the law of mass conservation for the gas in the controlled volume by way of equation of the air motion mechanical energy and work for overcoming of the frictional force, the second airflow during the pumping-out of the vacuum system will be [13]:

$$
m=S_{c s a} \cdot \sqrt{\frac{2 \cdot g}{1+\xi} \cdot p_{1} \cdot \rho_{1} \cdot\left(x^{\frac{2}{n}}-x^{\frac{n+1}{n}}\right)}
$$

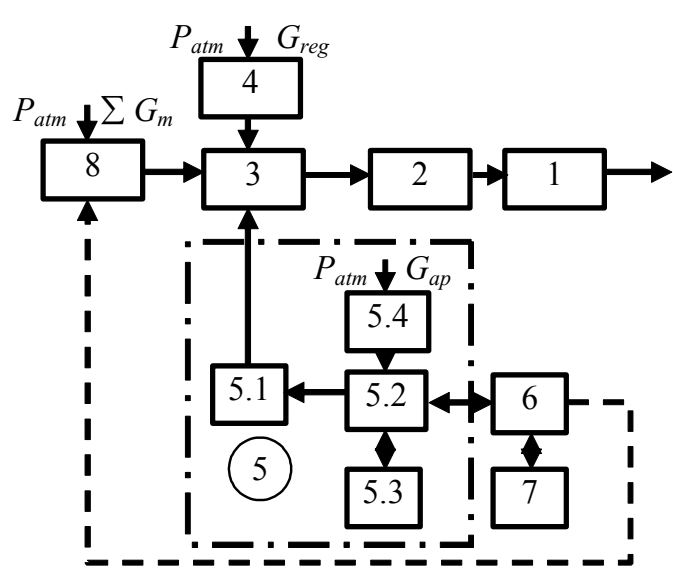

Fig. 1. The structure and functionally scheme of the vacuum system of milking machine: 1 - vacuum pump; 2 - vacuum reservoir; 3 - vacuum hose; 4 - vacuum gage pressure regulator; 5 - pulsator of the milking machine; 5.1 - chamber of the constant vacuum gage pressure; 5.2, 5.3 - chambers of the variable pressure; 5.4 - chamber of the atmospheric pressure; 6 - controller of the milking machine; 7 - interwall chamber of the teet cup; 8 - milk pipeline.

Differential equation of the $V$ air volume pumpingout at the condition of $M$ air mass by the $\tau$ time equals to $d M=V \cdot d \rho$ the mass increase [14]:

$$
d M=S_{c s a} \cdot \psi \cdot \sqrt{p_{i} \cdot \rho_{i}} \cdot d \tau,
$$

where $\psi=\sqrt{\frac{2 \cdot g}{1+\xi} \cdot\left(x^{\frac{2}{n}}-x^{\frac{n+1}{n}}\right)}-$ velocity aspect ratio defines the pressure relation at the air pumping-out, $x-$ ratio of the current value of the vacuum gage pressure to the atmospheric pressure, $x=p_{i} / p_{A}, p_{i}$ i $\rho_{i}$-current value of the pressure and the density in the volume $V$.
Taking into account that $\rho_{i}=\rho_{A} \cdot\left(\frac{p_{i}}{p_{A}}\right)^{\frac{1}{n}}$, the equation

(10) will have the appearance:

$$
\begin{aligned}
& d M=S_{c s a} \cdot \psi \cdot \sqrt{p_{i} \cdot \rho_{A} \cdot\left(\frac{p_{i}}{p_{A}}\right)^{\frac{1}{n}}} \cdot d \tau= \\
& =S_{c s a} \cdot \psi \cdot \sqrt{p_{A} \cdot \rho_{A}} \cdot \sqrt{\left(\frac{p_{i}}{p_{A}}\right)^{\frac{n+1}{n}}} \cdot d \tau
\end{aligned}
$$
have:

Also taking into account that $d M=V d \rho_{i}$, we will

$$
d M=V \cdot \rho_{A} \cdot d\left[\left(\frac{p_{i}}{p_{A}}\right)^{\frac{1}{n}}\right]=\frac{V}{n} \cdot \rho_{A} \cdot\left(\frac{p_{i}}{p_{A}}\right)^{\frac{1-n}{n}} \cdot d\left(\frac{p_{i}}{p_{A}}\right)^{.}
$$

After the dependences of (11) and (12) were equated, the sign was accounted and formula was reduced by $\left(p_{i} / p_{A}\right)^{1 / 2}$, the differential equation of air pumping out from the $V$ volume will have the appearance:

$$
\begin{aligned}
& \rho_{A} \cdot \frac{V}{n} \cdot\left(\frac{p_{i}}{p_{A}}\right)^{-1} \cdot d\left(\frac{p_{i}}{p_{A}}\right)= \\
& =-S_{c s a} \cdot \psi \cdot \sqrt{p_{A} \cdot \rho_{A}} \cdot \sqrt{\left(\frac{p_{i}}{p_{A}}\right)^{\frac{n-1}{n}}} \cdot d \tau
\end{aligned}
$$

The (13) formula is transformed and integrated with the limitations from 1 to $p_{i} / p_{A}$ and from 0 to $\tau$ :

$$
\frac{1}{n} \cdot \int_{1}^{\frac{p_{i}}{p_{A}}}\left(\frac{p_{i}}{p_{A}}\right)^{\frac{1-3 n}{2 n}} \cdot d\left(\frac{p_{i}}{p_{A}}\right)=S_{c s a} \cdot \psi \cdot \frac{1}{V} \cdot \sqrt{\frac{p_{A}}{\rho_{A}}} \cdot \int_{0}^{t} d \tau \cdot
$$

The integration results define the duration of air pumping out of the $V$ volume from the pressure $p_{A}$ to the vacuum gage pressure $p_{i}$, and will be determined in series by the formula:

$$
\begin{gathered}
\frac{\left.\left(\frac{p_{i}}{p_{A}}\right)^{\frac{1-n}{2 n}}\right|_{\frac{p_{i}}{p_{A}}} ^{n}}{\frac{1-n}{2 n}}=\left.S_{c s a} \cdot \psi \cdot \frac{1}{V} \cdot \sqrt{\frac{p_{A}}{\rho_{A}}} \cdot \tau\right|_{0} ^{\tau}, \\
\tau=\frac{2}{1-n} \cdot \frac{V}{S_{c s a} \cdot \psi} \cdot \sqrt{\frac{\rho_{A}}{p_{A}}} \cdot \sqrt{\left(\frac{p_{i}}{p_{A}}\right)^{\frac{1-n}{n}}}-1
\end{gathered}
$$

To simulate the characteristic of the vacuum gage pressure changes in the vacuum system of the milking machine one has to know the time parameters of the vacuum pressure - the time interval of the air pumping out from the volumes of the variable vacuum gage pressure. From the equation (16) the dependences of pressure changes in time have been derived: 


$$
p_{i}=p_{A} \cdot \sqrt[1-n]{\frac{(\tau+1)^{2 \cdot n} \cdot(1-n)^{2 \cdot n} \cdot S_{c s a}^{2 \cdot n} \cdot \psi^{2 \cdot n} \cdot p_{A}}{4^{n} \cdot V^{2 \cdot n} \cdot \rho^{n}}}
$$

where $V$ - the volume of chambers of the variable vacuum gage pressure of the milking machine, $\mathrm{m}^{3}$, $S_{c s a}$ - the cross-sectional area of the pulsator drain port, $\mathrm{M}^{2}, \psi$ - velocity aspect ratio defines the pressure relation, $\mathrm{m}^{1 / 2} / \mathrm{s}, \rho_{A}$ - the air density in the $V$ volume for the atmospheric pressure, $\mathrm{kg} / \mathrm{m}^{3}, p_{A}$ - the air atmospheric pressure in the $V$ volume, $\mathrm{kg} / \mathrm{m}^{2}, p_{i}$ - the value of the vacuum gage pressure in the $i$-th point of time, $\mathrm{kg} / \mathrm{m}^{2}, n-$ polytropic coefficient, $n=1,41$.

Thus, the quantity of pumped out air will be:

$$
G_{i}=\frac{p_{i} \cdot V}{R_{n} \cdot \Theta}
$$

where $V$ - the volume of chambers of the variable pressure, $\mathrm{M}^{3}, \quad R_{n}$ - gas constant of the air, $R_{n}=287,72 \mathrm{~J} /\left(\mathrm{kg} \cdot{ }^{\circ} \mathrm{C}\right), \Theta-$ air temperature, ${ }^{\circ} \mathrm{C}$.

For connection of the $i$ chambers with the given volume one can determine the volume, pressure and the air quantity accordingly [12]:

$$
V=\sum_{i=1}^{n} V_{i}, \quad p_{V}=\frac{1}{n} \sum_{i=1}^{n} p_{i}, \quad G=\sum_{\tau=0}^{1} G_{i} \cdot
$$

The amplitude of the vacuum gage pressure oscillation in the vacuum pipeline is determined by the formula:

$$
\Delta p_{V P}=\frac{R_{n} \cdot \Theta \cdot\left(G_{V P}+G+G \cdot\left(N_{n m u}^{+}-1\right) \cdot P\left(N_{n m u}\right)\right)}{V_{V P}},
$$

where $G_{V P}, G$ - the air quantity in vacuum pipeline and volumes of the variable vacuum gage pressure accordingly, $\mathrm{kg}, V_{V P}$ - the volume of the vacuum pipeline of the specific milking machine, $\mathrm{m}^{3}, P\left(N_{n m u}\right)-$ the probability of simultaneity and coincidence of the phase and work times of the specific milking machines:

$$
P\left(N_{n m u}\right)=P\left(N_{n m u}^{+}\right) \cdot P\left(N_{n m u}^{-}\right),
$$

where

$$
P\left(N_{n m u}^{+}\right)=\frac{a_{1}^{N_{m m u}}}{N_{n m u}^{+} \downarrow} \cdot \mathrm{e}^{-a 1}-\text { the probability of }
$$

simultaneity and coincidence of the phase and work times of the milking machines according to the Poisson limit theorem [15],

$$
P\left(N_{n m u}^{-}\right)=\frac{a_{2}^{N_{\text {max }}}}{N_{n m u}^{-} \downarrow} \cdot \mathrm{e}^{-a 2}-\text { the }
$$

probability of unsimultaneity and uncoincidence of the phase and work times of the milking machines according to the Poisson limit theorem [15], $a_{1}=n_{m u} \cdot p\left(_{n m u}^{+}\right)$, $a_{2}=n_{m u} \cdot p\left(_{n m u}^{-}\right), \quad n_{m u}-$ the quantity of the simultaneously working milking machines, pcs., $N_{n m u}^{+}-$ the quantity of the simultaneously working milking machines with the coincidence in time of the phase and work times of pulsator, pcs., $N_{n m u}^{-}-$the quantity of the simultaneously working milking machines with the uncoincidence in time of the phase and work times of pulsator, pcs., $p\left({ }_{n m u}^{+}\right)$- probability of the coincidence in time of the phases and work times of pulsator for $N_{n m u}^{+}$ simultaneously working milking machines, $p\left(_{n m u}^{+}\right)=N_{n m u}^{+} / n_{m u}, \quad p\left(\left(_{n m u}^{-}\right)^{-}\right.$probability of the uncoincidence in time of the phases and work times of pulsator for $N_{n т u}^{-}-$simultaneously working milking machines, $p\left(_{n m u}^{-}\right)=N_{n m u}^{-} / n_{m u}$.

\section{Results}

The productivity of the milking machine (equation 8) depends on the duration of preparation $t_{p}$ and final $t_{f}$ operations, duration of machine milking $t_{m m}$ and the cow productivity. Also the productivity of the milking machine depends of the quantity of the milking units, which are operated by the MMO with the regulated load factor $K_{M M O}$. The results of the modelling are shown in Fig. 2 and 3.

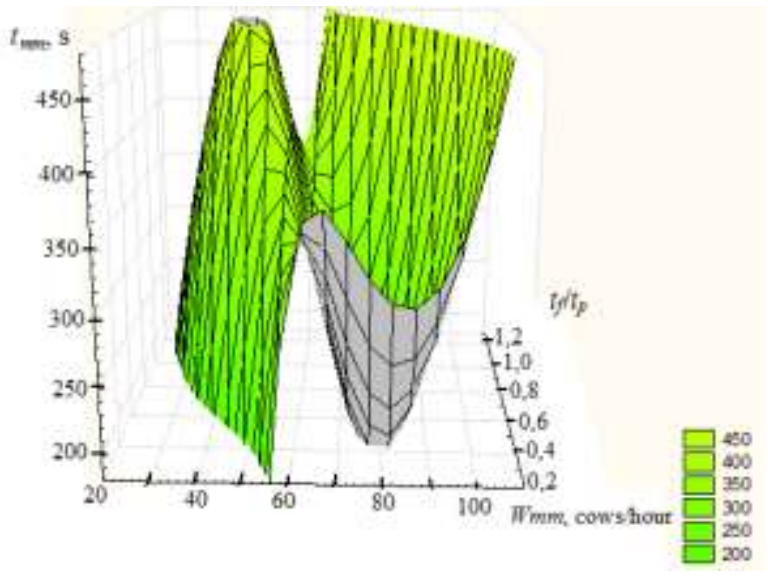

Fig. 2. Dependence of the productivity of the milking machine from the duration $t_{m m}$ of machine milking of cow and ratio of $t_{f} / t_{p}$, by the $t_{f} \rightarrow \max , t_{p} \rightarrow \max$.

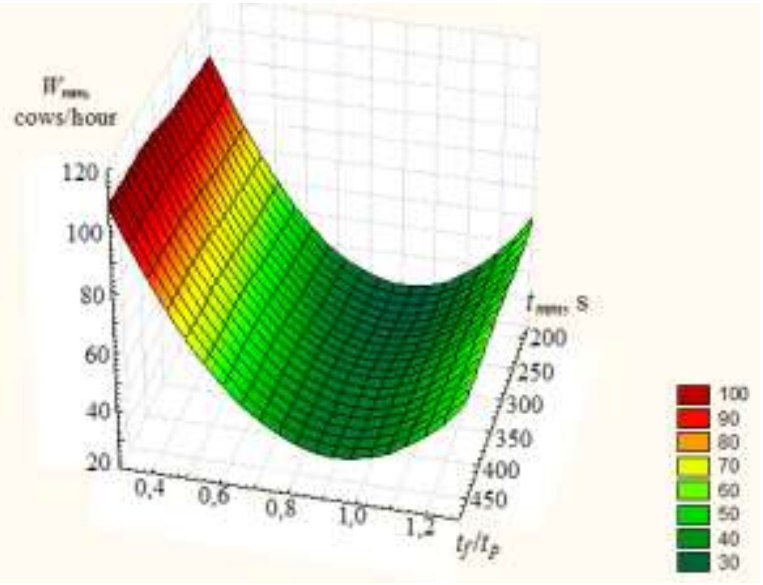

Fig. 3. Dependence of the productivity of the milking machine from the time $t_{m m}$ of machine milking of cow and ratio of $t_{f} / t_{p}$, by the $t_{f} \rightarrow \min , t_{p} \rightarrow \min$

The analysis of dependence of the productivity of the milking machine from the duration $t_{m m}$ of machine milking of cow and ratio of $t_{f} / t_{p}$ (Fig.3) demonstrates 
when $t_{f} \rightarrow \max , t_{p} \rightarrow \max$, the productivity of the MMO work is not submitted to the specific law. The area on the 3 -D graph of the $26 \ldots 34$ cows/hour productivity fits with the milking machine for the bucket milking, 34...50 cows/hour - pipeline milking in the barn, from 51 cows/hour - parlor milking.

The analysis of dependence of the productivity of the milking machine from the time $t_{m m}$ of machine milking of cow and ratio of $t_{f} / t_{p}$ (Fig.2) demonstrates when $t_{f} \rightarrow \min , t_{p} \rightarrow \min$, the productivity of the MMO work is submitted to the second-order equation.

The area on the 3-D graph of the $29 . . .36$ cows/hour productivity fits with the milking machine for the bucket milking, $37 . .50$ cows/hour with the pipeline milking in the barn, from the 51 cows/hour and high with the parlor milking.

Modelling of the pressure oscillation amplitude in the vacuum pipeline is demonstrated in Fig. 4.
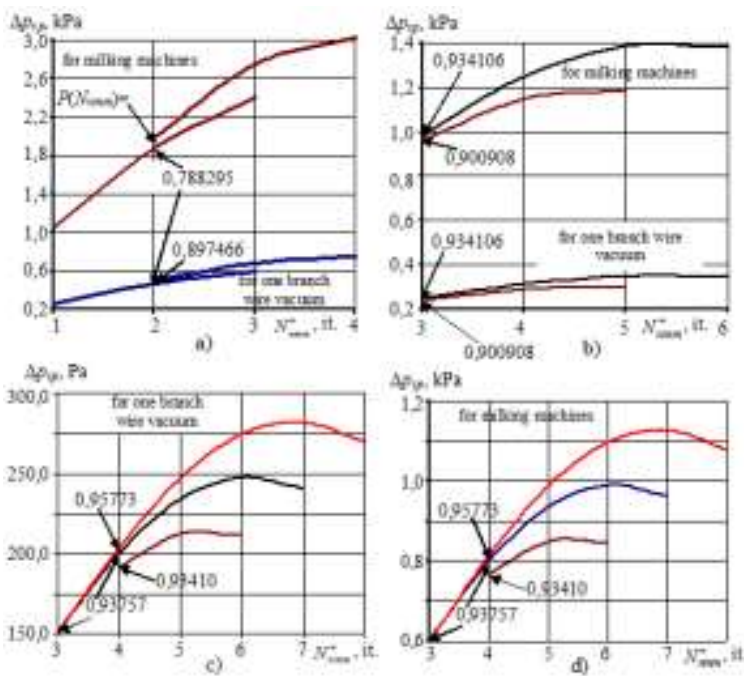

Fig. 4. Graphs of modelling of the vacuum gage pressure oscillation $\Delta p_{v p}$ in the vacuum pipeline of milking machine in dependence of quantity of the milking units $N_{n m u}^{+}$with the

coincidence in time of the phase and work times of pulsator with probability of $P\left(N_{n m u}\right)$ for diameter of vacuum pipeline $D$ : $\mathrm{a}-D=25,4 \mathrm{~mm}$, milking unit with pneumatic pulsator; $\mathrm{b}-$ $D=40 \mathrm{~mm}$, milking unit with pneumatic and electric pulsator of the pair-wise action; c, $\mathrm{d}-D=50 \mathrm{~mm}$, milking unit with pneumatic and electric pulsator of the pair-wise action.

Analysis of results of the vacuum pressure oscillations modelling shows that the maximum oscillations amplitude of vacuum pressure at a frequency of $1 \mathrm{~Hz}$ does not exceed $\Delta p_{v p}=1.4 \mathrm{kPa}$ for vacuum pipeline of $\mathrm{D}=40 \mathrm{~mm}$ diameter with a probability of $P\left(N_{n m u}\right)=0.76212$. To vacuum pipeline with diameter of $D=25.4 \mathrm{~mm}$ the maximal oscillations amplitude of vacuum pressure is $\Delta p_{v p}=3.03 \mathrm{kPa}$ with probability of $P\left(N_{\text {nти }}\right)=0.628837$.

For the number of milking machines which are working synchronous with the maximum probability of coincidence in time cycles and phase of pulsators work the vacuum pressure oscillations does not exceed $\Delta p_{v p}=2 \mathrm{kPa}$. For the vacuum pipeline of $D=50 \mathrm{~mm}$ diameter the vacuum pressure oscillations are
$\Delta p_{v p}=800 \ldots 812 \mathrm{~Pa}$ with the maximum probability of $P\left(N_{n m u}\right)=0.93757 \ldots 0.95773$.

Graphic dependence of the vacuum pressure oscillation amplitude changes $\Delta p_{v p}$ from the number of simultaneously working milking machines $N_{n m u}^{+}$with coincidence in time of the cycles and phases of pulsator work with probability $P\left(N_{n m u}\right)$ and the vacuum pipeline $\mathrm{D}$ diameter was shown in Fig. 5.

The analysis of simulation results shows that with an increase of the number of milking machines the vacuum pressure amplitude is increased. From $N_{n m u}^{+}=8$ with the $n_{m u}=8$ probability of coincidence in time of the pulsator cycles and phases of $P\left(N_{n m u}\right)=0.592547$ the oscillations amplitude of vacuum pressure will be $\Delta p_{v p}=4.72 \mathrm{kPa}$ for the diameter of milking machines vacuum pipeline of $D=25.4 \mathrm{~mm}$. For the vacuum pipeline diameter of $D=50 \mathrm{~mm}$ and the same number of milking machines the amplitude of vacuum pressure oscillations will be $\Delta p_{v p}=1.08 \mathrm{kPa}$.
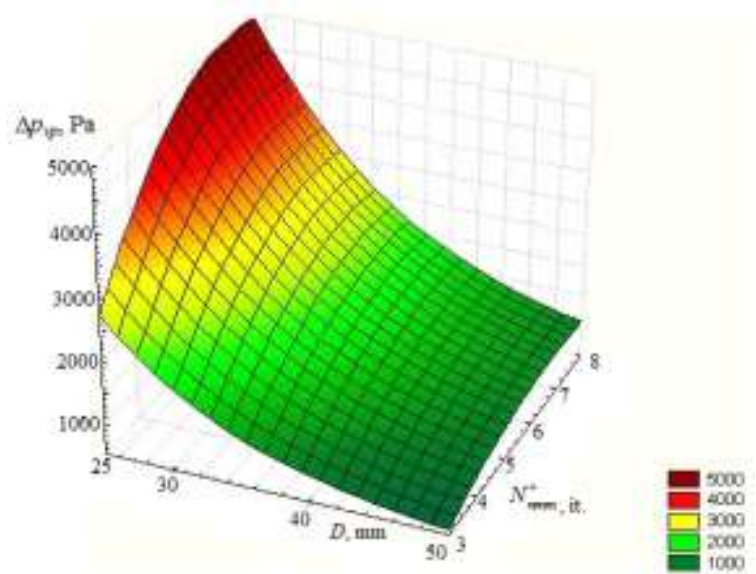

Fig. 5. The dependence of change of the $\Delta p_{v p}$ vacuum pressure amplitude oscillation from the number of simultaneous working milking machines $N_{n m u}^{+}$with coincident in time of pulsator cycles and phases and $D$ machine vacuum pipeline diameter .

The maximum probability of coincidence in time of the pulsators cycles and phases is $P\left(N_{n m u}\right)=0.95773$ for $N_{n m u}^{+}=4$ machines, then the amplitude of vacuum pressure oscillations will be equal to $\Delta p_{v p}=3.55 \mathrm{kPa}$ per one cycle of pulsator work (frequency of $1.0 \mathrm{~Hz}$ ) for milking machines vacuum pipeline diameter of $D=25.4 \mathrm{~mm}$ and $\Delta p_{v p}=0.81 \mathrm{kPa}$ for $D=50 \mathrm{~mm}$.

\section{Discussion and conclusion}

The dependences (1-5) show that efficiency depends on the parameters of the technical system which have to provide the conditions for the milk take out from the cow udder in accordance with the physiology of milk lactogenesis and ejection with reduction of both the MMO labor inputs and energy expense in the process of milking machine system functioning.

The requirements of the machine milking process were fulfilled for the $K_{M M O} \geq 1$. The milking duration in 
the ranging ratio of $t_{f} / t_{p}=0.5 \ldots 0.8$ are reduced due to the optimal number of milking machines which are operated by $\mathrm{MMO}$ with the $K_{M M O} \approx 1$. At the conditions of $K_{M M O}<1$ the MMO has no enough time to fulfil all operations by the requirements of the machine milking process due to unreasonable number of operated milking machines.

It can be noted that the maximum vacuum oscillation in milking machines vacuum pipeline is not more for the permissible parameters $\left(\Delta p_{v p} \leq 2.5 \mathrm{kPa}\right)$ at the milking in the cattle barn with a frequency of $1 \mathrm{~Hz}$. With the diameter of vacuum pipeline increasing the amplitude of vacuum gage pressure oscillation is decreased.

The analysis and the theoretical studies of technological process of the cow machine milking make possible to define the requirements for the improvement of technological process and technical means with the efficiency rise of milking systems in the following way: - to provide a flexible relationship amongst the intensity of milk ejection and constructive and technological parameters of milking machine systems, to provide the adaptation of the technical system into the function of milk ejection of each individual cow;

- to create of cyber-physical milking machine that would conform with the physiological characteristics of cows as to the milk ejection;

- to optimize of the organizational and technological and structural energetic parameters of technological process and milking machines for adaptation both the technical system and the physiological state of the cow organism.

Using of cyber-physical systems of cows machine milking increases the milking machines productivity by the $1.26 \ldots 1.85$ times.

For the vacuum pressure oscillations of $\Delta p_{v p}=2500 \mathrm{~Pa}$ the suction ability of milking machines for modes will be accordingly of $E=4.093, E_{1}=4.177$, $E_{2}=4.007 \mathrm{~m} / \mathrm{s}$. For the milking machine phase proportions were taken the standard value. Accordingly the reducing of milk yields per cow in the energy equivalent is $65.1 \mathrm{MJ}(3.08 \mathrm{MJ} / \mathrm{kg})$. The energy equivalent of milk production will be $11450 \mathrm{MJ}$ accordingly. The energy expenses of milking machine for the year in energetic equivalent will be $16556.4 \mathrm{MJ}$. The efficiency index of conventional milking machine will be $K_{B T S 1}=46.6$. According to the results of studies of the technological process of milking, the $450 \mathrm{~W}$ $(1.62 \mathrm{MJ})$ reducing of the vacuum machine drive power will enable annual saving of $1773.9 \mathrm{MJ}$. Accordingly in practice one can use the $1 \mathrm{MMO}$ less with saving in the energy equivalent of $1.971 \mathrm{MJ}$. Then the efficiency index of adapted systems functioning of the milking machines will be $K_{B T S 2}=55.3$. Accordingly, the efficiency index of the milking machines systems functioning will increase by $18.7 \%$.

\section{References}

1. V. T. Dmytriv, New direction of development of the technologies and technical means in dairy cattle husbandry: Materials of the 13th International Symposium on Machine Milking of Farm Animals,
27-29 of June, 2006. Milking machine with microprocessor control, 161-165 (2006).

2. V. T. Dmytriv, Mechanization and Electrification of Agriculture, Interdepartmental special scientific coll. Hlevakha, Automated milking machine, 84, 134-136 (2010)

3. V. T. Dmytriv, Mechanization in Agriculture. International Scientific, Scientific Applied and Informational Journal, Sofia, Adaptive machine milking system, 10, 15-18 (2015)

4. V. T. Dmytriv, Mechanics and Technology Bases of the Milking Machine Systems. Theory and Practice: Monograph (2017)

5. V. Dmytriv, V. Adamchuk, I. Dmytriv, MOTROL. Commission of Motorization and Energetics in Agriculture, Adaptive machine milking with the pneumatic electric magnetic pulsator, 17(9), 83-87 (2015)

6. A. Pazzona, L. Murgia, L. Zanini, et all. ASAE Annual International Meeting, Riviera Hotel and Convention Center, Las Vegas, Nevada, USA, Dry tests of vacuum stability in milking machines with conventional regulators and adjustable speed vacuum pump controllers (2003)

7. V. Dmytriv, MOTROL. Commission of Motorization and Energetics in Agriculture, Analytical study of the pressure regulator for pulsating vacuum systems, 18/8, 69-76 (2016)

8. D. J. Reinemann, N. Schuring, R. D. Badel, The Sixth International ASABE Dairy Housing Conference, Minneapolis, Minnesota, USA, Methods for Measuring and Interpreting of the Milking Vacuum (2007)

9. L.P. Kartashov, Methods for calculating of the biological and technical parameters of the "humanmachine-animal” system, Orenburg: OSAU Publishing Center (2007)

10. V. T. Dmytriv, Research Journal of National University of Life and Environmental Sciences of Ukraine: Technics and Energy of Agriindustrial Complex, Modeling of the number of milking machines at the optimum workload of the milking machine operator, 212/2, 167-173 (2015)

11. V. T. Dmytriv, Bases of the Theory of the Machinery Usage in the Animal Husbandry, Lviv : Afisha (2008)

12. V. Dmytriv, Journal of Lviv National Agrarian University : Agrarian Engineer Research, Model of the air flow by the elements of the milking machine, 10, 483-488 (2006)

13. I. V. Dmytriv, V. T. Dmytriv, Research Journal of the Kharkiv Petro Vasylenko National Technical University of Agriculture "Technical Systems and Technologies of the Animal Husbandry", Model of air pumping from the chambers of variable vacuum gage pressure of the milking machine, 132, 207-212 (2013) 
14. V. Dmytriv, I. Dmytriv, MOTROL. Commission of Motorization and Energetics in Agriculture, Modeling of the time of air outflow from the bounded space, 15/4, 193-197 (2013)

15. P. F. Ovchinnikov, B. M. Lisitsin, V. M. Mikhailenko, Higher Mathematics, Kyiv : Higher school, (1989) 